\title{
EL COMPLEJO DE ADONIS: MASCULINIDAD E IMAGEN CORPORAL ${ }^{1}$
}

\author{
Carlos Antonio Rivera Otero ${ }^{2}$
}

\section{Resumen}

Este artículo discute el tema de los problemas de imagen corporal en hombres. Específicamente los relativos a la investigación clínica en progreso sobre la Dismorfia Muscular (Complejo de Adonis). Se presentan los aspectos culturales relativos a la situación y los efectos en la salud física y mental de conductas asociadas como el uso de esteroides anabólicos. Se aborda la situación desde el marco conceptual de la Perspectiva de Género y la Construcción Social de la Masculinidad. Se presentan los resultados de una investigación realizada a 20 hombres que asistían al gimnasio a realizar ejercicios en la Zona Metropolitana de San Juan. Está investigación exploró la motivación para ir al gimnasio a realizar ejercicios y la percepción en torno a la imagen corporal. Finalmente se discuten las implicaciones para la práctica del Trabajo Social.

Descriptores: Imagen corporal, dismorfia muscular, esteroides anabólicos, masculinidad y masculinidad hegemónica.

\section{Abstract}

This article discusses the theme of masculine body image problems. Specifically, in progress clinical research of Muscular Dysmorphia (Adonis Complex). It displays the cultural aspects of the problem and the effects of the associated behaviors like the use of anabolic steroids in physical and mental health. The author addresses the situation from the conceptual framework of Genders Perspective and the Construction of Masculinity. Discuss the findings of research accomplished with 20 men that attend to a gym at the Metropolitan Area of San Juan, Puerto Rico. The research explored the motivation of men to exercise and the perception they had of their body image. Finally it discusses the implications for the profession of Social Work.

\footnotetext{
${ }^{1}$ Este artículo está basado en la tesis de maestría de la Escuela Graduada de Trabajo Social, Universidad de Puerto Rico en Río Piedras.

${ }^{2}$ Trabajador Social de la Unidad de Gestión de Calidad y Adiestramiento en la Región San Juan del Departamento de la Familia; Profesor en el Programa de Trabajo Social de la Universidad del SagradoCorazón, Santurce y Universidad del Este, Carolina.
} 
Keywords: Body image, muscular dysmorphia, anabolic steroids, masculinity, hegemonic masculinity.

\section{Introducción}

Una de las características de las sociedades modernas es la preocupación por la imagen corporal (Baile, Monroy y Garay, 2005). Esta situación crea una presión social que se traduce en un amplio mercado de productos para el adelgazamiento, incremento en la actividad deportiva sin mesura ni supervisión, proliferación de gimnasios y miles de dietas para perder peso. Es en la articulación de estos factores sociales que se ubican las alteraciones relativas a la imagen corporal en hombres.

La imagen corporal es un constructo psicosocial complejo, que se refiere a cómo la autopercepción del cuerpo se articula en una representación mental, compuesta por un esquema corporal perceptivo con emociones, pensamientos y conductas asociadas (Baile Ayensa, 2002). Con relación a la imagen corporal masculina podríamos referirnos al conjunto de descripciones, pensamientos y sentimientos que tienen los hombres con respecto a la forma y tamaño de su cuerpo y en específico de sus músculos.

Existe una extensa literatura sobre el tema de la insatisfacción con la imagen corporal en mujeres. Sin embargo el estudio de la imagen corporal en hombres es un área de investigación novel y poco explorada. Rutsztein, Casquet, Leonardelli, López, Macchi, Marola y Redondo (2004) establecen que son pocos los estudios internacionales publicados sobre el tema. Baile, Monroy y Garay (2005) añaden que en los países iberoamericanos no se dispone de investigaciones epidemiológicas de los trastornos de la imagen corporal masculina. Bergstrom \& Neighbors (2006) argumentan que la investigación en esta área evidencia que los hombres y las mujeres son similares en términos de los disturbios de imagen corporal. Muchos hombres al igual que muchas mujeres están insatisfechos con alguna parte de sus cuerpos.

Rutsztein et al. (2004) argumentan que la evaluación sobre la preocupación por la imagen corporal en hombres y las conductas asociadas, ha surgido como un tema importante de investigación. La mayoría de los trabajos publicados han encontrado asociación entre la práctica excesiva de ejercicios en los gimnasios, la distorsión de la imagen corporal y alteraciones en los patrones de alimentación. Según Baile (2003) varias investigaciones han encontrado que los hombres que practican ejercicios en gimnasios tienen más problemas relativos a su imagen corporal que los hombres que no acuden al mismo. Pope, Phillips y Olivardia (2000) expresan que muchos de estos hombres tienen una percepción irreal de su apariencia y es por esta razón que recurren al uso y abuso de drogas ergogénicas, ejercicios excesivos y gastan billones de dólares en suplementos dietéticos anualmente, los cuales en muchas ocasiones tienen efectos colaterales en la salud física y mental. Facchini añade que los adolescentes y los adultos jóvenes están tomando medidas drásticas hacia sus cuerpos. Señala que el 
mismo sistema económico y social que ha explotado la experiencia corporal de las mujeres se está enfocando en los hombres.

Un creciente número de hombres están tomando medidas con respecto a su imagen corporal realizando levantamiento de pesas de forma compulsiva, usando esteroides anabólicos, realizándose cirugía estética, entre otras prácticas. De acuerdo a Pope et. al (2000) este comportamiento en ocasiones, está cruzando la línea de un interés generalmente aceptable por el cuidado y la imagen corporal a una obsesión patológica. La autora y los autores citados han nombrado esta nueva obsesión con la apariencia física y corporal masculina como el Complejo de Adonis.

En los primeros años de la década de 1990, en el Laboratorio de Psiquiatría Biológica del Hospital Mc Lean, Harrison Pope y sus colaboradores observaron 108 físiculturistas, entre los cuales había nueve individuos que expresaban tener un cuerpo pequeño y poco musculoso, sin embargo, se observaban fuertes y con grandes músculos. Estos hombres informaron que en muchas ocasiones evitaban situaciones sociales, lugares o utilizaban ropas encubridoras en colores y tamaño por temor a que se vieran poco musculosos. En ocasiones en que la exposición corporal era inevitable vivían el momento con gran angustia, ansiedad y frustración (Facchini, 2006).

El grupo de investigadores Pope, Katz \& Hudson (1993) Olivardia, Pope \& Hudson (2000) inicialmente describieron estas preocupaciones y conductas con relación a la imagen corporal como un síndrome al que nombraron Anorexia Inversa. A partir de la publicación del informe inicial a principios de los años de la década de 1990, los investigadores e investigadoras continuaron encontrando más hombres que realizaban ejercicios que reportaban una preocupación con la noción de que no eran suficientemente musculosos. Algunos informaron serios deterioros en su funcionamiento social y laboral.

Con el desarrollo de la investigación Pope y sus colaboradores modificaron el término de Anorexia Inversa a Vigorexia y finalmente a Dismorfia Muscular por considerar que estaba relacionado con el Trastorno Dismórfico Corporal según establecido en el DSM IV (Pope, Gruber, Choi, Olivardia y Phillips, 1997). El Trastorno Dismórfico Corporal según Facchini (2006), es una entidad siquiátrica definida en el Manual Diagnóstico y Estadístico de Desórdenes Mentales conocido regularmente como el DSM IV R (APA, 2000). El trastorno Dismórfico Corporal (TDC), está incluido dentro de los Trastornos Somatomorfos.

La Dismorfia Muscular aparenta representar una forma del Trastorno Dismórfico Corporal (TDC), excepto que está caracterizado por una preocupación por la muscularidad total del cuerpo, en oposición con alguna anomalía física, según especifican los criterios diagnósticos del Trastorno Dismórfico Corporal (TDC). No obstante, Arbinaga y Caracuel (2003) expresan que no existe un modelo etiológico que permita explicar las 
condiciones para la aparición de la Dismorfia Muscular, más bien con lo que se cuenta es con especulaciones de diversas posibilidades. Destacan, sin embargo, que se busca ajustar las hipótesis etiológicas al Modelo Biopsicosocial (Pope et al. 1997; Olivardia et al. 2000 y Pope et al., 2000). La Dismorfia Muscular ha sido catalogada en algunos casos como parte del espectro de los Desórdenes Obsesivo Compulsivos.

Como hasta el presente los manuales relacionados a diagnósticos de salud mental no han reconocido la Dismorfia Muscular como parte de algún trastorno, Pope et al. (1997) han propuesto una serie de criterios diagnósticos para la Dismorfia Muscular, los cuales detallaremos a continuación:

- Preocupación por no ser suficientemente magro (libre de grasa) y musculoso. Las conductas características asociadas incluyen varias horas de levantar pesas y excesiva atención a la dieta.

La preocupación se manifiesta en al menos dos de los siguientes cuatro criterios:

- El individuo frecuentemente abandona actividades sociales, laborales o recreativas importantes por una necesidad compulsiva de mantener su esquema de trabajo muscular y dieta.

El individuo frecuentemente abandona actividades sociales, laborales o recreativas importantes por una necesidad compulsiva de mantener su esquema de trabajo muscular y dieta.

- El individuo evita situaciones donde su cuerpo resulta expuesto a otras personas o soporta dichas situaciones con un marcado malestar y ansiedad intensa.

- La preocupación por ser poco musculoso o corpulento causa malestar clínico significativo o interfiere en su funcionamiento social, laboral u otras áreas importantes.

- El individuo continúa entrenando, haciendo dieta o utilizando sustancias ergogénicas (aumentadoras del rendimiento físico) a pesar de conocer las consecuencias adversas físicas o psicológicas.

El principal foco de la preocupación y conducta es en ser demasiado pequeño o inadecuadamente musculoso, que se diferencia del miedo a engordar de la anorexia nerviosa o a una preocupación primaria solamente con otros aspectos de la apariencia como en tras formas de Trastorno Dismórfico Corporal. En términos diagnósticos los autores y la autora citados consideran que la Dismorfia Muscular está relacionada al Desorden Obsesivo-Compulsivo. Los investigadores y la investigadora creen muy importante no dejar fuera otras variables como son la enorme presión social para que los hombres luzcan musculosos y magros, al igual que pre-disposiciones biológicas con ciertos trastornos mentales como puede ser el Obsesivo Compulsivo. La presión social puede desarrollar en algunos hombres síntomas profundos que pueden afectar su salud integral. 


\section{Aspectos culturales relativos a la Dismorfia Muscular}

En cuanto a los aspectos sociales, culturales e históricos con relación a los ideales corporales masculinos Facchini (2006) argumenta que los mismos han incrementando en muscularidad con el pasar de las décadas. Las estrellas de cine de las películas de Hollywood en los años 50 y 60 no tenían la misma masa muscular de los héroes de las películas que se desarrollaron en las décadas de los 80 y 90 (Pope et al., 2000).

Los niños reciben mensajes sobre los ideales de imagen corporal desde muy temprano en su infancia. Los muñecos de acción han moldeado la imagen corporal masculina en los niños varones a través de las décadas. Pope, Olivardia \& Boroweicki (1999) realizaron un estudio obteniendo muestras de las figuras de acción más populares en los Estados Unidos en los últimos treinta años anteriores al estudio. Midieron la circunferencia del pecho, los bíceps y la cintura de los juguetes. Estas medidas fueron transferidas a la escala métrica en proporción a la altura promedio de un hombre actual. Los resultados obtenidos indicaron que las figuras de acción habían crecido en masa muscular excediendo la muscularidad de los más grandes levantadores de pesas actuales.

Los mensajes culturales respecto a la imagen corporal continúan mientras los varones transitan de la etapa de la niñez a la adolescencia. Los investigadores e investigadoras citados destacan el interés de los adolescentes en la lucha libre. En la actualidad podemos observar los juegos electrónicos en los cuales los héroes y villanos que se enfrentan poseen dimensiones musculares impresionantes e inalcanzables.

La publicidad juega un papel importante en la transmisión de los mensajes relativos a los ideales de imagen corporal masculinos. Leit, Pope y Gray (2001), argumentan que el mismo tipo de publicidad que por mucho tiempo persiguió a las mujeres con ideales de delgadez inalcanzables se enfoca en nuestros días en la población masculina incluyendo niños, adolescentes y adultos jóvenes. Las imágenes masculinas en la publicidad han aumentado su definición, tono muscular y el tamaño de sus genitales (Facchini, 2006). Los medios masivos de comunicación por medio de la publicidad crean una representación de la imagen corporal masculina ideal y socialmente aceptable. Esto contribuye a un aumento de la insatisfacción corporal en algunos hombres impactados por estas fuerzas mediáticas (Leit, Gray \& Pope,2002). La tendencia de la publicidad comercial llevó a Pope et al. (2000), a considerar que el cuerpo masculino está creciendo como una marca distintiva de la masculinidad. La preocupación de los hombres con su imagen corporal iría aumentando junto con implicaciones clínicas y socio-culturales. Entre estas implicaciones se destacan la proliferación de gimnasios, la aparición de la Dismorfia Muscular en hombres pre-dispuestos, la utilización de suplementos dietéticos y el uso de esteroides anabólicos. 


\section{Uso de esteroides anabólicos}

Los esteroides anabólicos androgénicos son una versión sintética de la hormona masculina testosterona. La palabra esteroides está relacionada a su carácter hormonal y anabólico a la construcción de tejido muscular. Androgénicos se refiere a la búsqueda de maximizar las características masculinas. Los esteroides anabólicos son utilizados para aumentar la masa muscular, para tener más resistencia y fortaleza (Irigoyen Coll, 2005). Forman parte de las drogas ergogénicas o de desempeño. Se pueden utilizar de forma oral o inyectable intramuscularmente.

Pope et al. (2000) describen el uso de esteroides anabólicos como un problema de salud pública. Indican que como problema de esta índole sería mínimo si sólo fueran unos cuantos profesionales del campo de los deportes y las artes cinematográficas quienes los utilizaran. Pero millones de adolescentes y hombres ven a estos atletas y artistas como modelos a seguir, y desean tener cuerpos como ellos. La insatisfacción con la imagen corporal en hombres es la fuerza poderosa que guía el mercado subterráneo de esteroides anabólicos. Como resultado de esto el uso y abuso de estas sustancias ha crecido, convirtiéndose en una de las manifestaciones más peligrosas del Complejo de Adonis.

En investigaciones que realizaron Kenayama, Pope, Cohane \& Hudson (2003) los resultados encontrados apuntaron a que los usuarios de esteroides anabólicos evidenciaron altos índices de uso, abuso y dependencia a otras sustancias ilegales que aquellos que no han utilizado esteroides anabólicos. De igual forma que el uso de estas drogas tiende a ocurrir en hombres con altos niveles de conducta antisocial y bajos niveles de estima con respecto a su cuerpo.

Con el uso de esteroides anabólicos androgénicos existe la posibilidad de daños a la salud física. Incrementan el riesgo de enfermedades del corazón, enfermedades del hígado, la posibilidad de cáncer prostático y ginecomastia. Irigoyen Coll (2005) apunta a la posibilidad de efectos adversos a nivel músculo-esquelético, desempeño sexual, capilar, hepático y renal. No obstante, el peligro mayor del uso de estos químicos, son los efectos a la salud mental que acarrean. Las personas que abusan de estas sustancias pueden desarrollar irritabilidad extrema llevándolos a episodios de violencia irracional y depresión cuando dejan de utilizarlos, lo que se conoce como síndrome de retirada (Pope et al. 2000). Algunos usuarios pueden tener episodios de violencia extrema en los que pueden llegar a cometer homicidios y suicidio. Estos episodios de violencia extrema son conocidos popularmente en el idioma ingles como "roid rage" (rabia o furia por esteroides). 


\section{Perspectiva de género y construcción social de la masculinidad}

La Perspectiva de Género, parte del reconocimiento de la subordinación social y política de las mujeres (Bonan y Guzmán, s.f.). Las perspectivas teóricas sobre género tienen sus raíces en el debate sobre la identidad, el poder y la articulación de la vida en los espacios, grupos e instituciones sociales. El análisis de la dominación masculina se centra en el concepto del Patriarcado. La estructura social del patriarcado tiene aproximadamente 5,000 años de existencia (Lerner, 1990). Este paradigma se basa en el sexismo, los privilegios masculinos en todos los ámbitos y se expresa en exclusión, misoginia y homofobia. El poder de género se refiere al dominio y subordinación de los hombres sobre las mujeres, los niños, las niñas y sobre otros hombres considerados débiles o menos hombres. Este poder se ejerce tanto en los espacios públicos (instituciones políticas y económicas) como en los espacios privados (vida familiar y estructuración de las tareas en el hogar).

Scott (1996) argumenta que el género es una creación social de ideas sobre los roles adecuados para hombres y mujeres. Es para esta autora una categoría social sobre un cuerpo sexuado. Lo masculino y lo femenino se representa como una dicotomía. En esta representación se adjudican relaciones de polaridad a características asociadas a los dos géneros. Las relaciones de polaridad planteadas se arraigan en los procesos de construcción de identidades en el ámbito individual mediante el proceso de socialización desde que somos niños y niñas. De igual forma lleva a que se construyan subjetividades colectivas y prescripciones de lo que debe ser un niño, una niña, una mujer y un hombre, con todo un imaginario sobre las formas, actitudes y conductas que cada cual debe asumir. La perspectiva de género nos remite como categoría central de análisis, a la construcción de las identidades masculinas y femeninas. En el presente articulo brindaremos atención primordial a la construcción de las identidades masculinas, ya que el problema de la Dismorfia Muscular es un problema principalmente masculino y que está conectado a la construcción de estas identidades.

La masculinidad es un discurso siempre cambiante de cómo ser hombre, cuya característica más destacada es que se iguala la masculinidad con la agresividad, la violencia, la competencia y la heterosexualidad. Este discurso es asumido por los hombres como un aspecto principal de su identidad. La masculinidad es un elemento primario de los privilegios asociados a los hombres. Aunque no todos los hombres los comparten, hay factores que influencian el acceso a estos privilegios como pueden ser la raza, la clase social u orientación sexual, entre otros. La masculinidad no es un fenómeno uniforme, estático e igualmente compartido por todos los hombres (Román, González, Fernández, Cruz y Ávila, 2003).

Badinter (1993) indica que no hay una masculinidad única, lo que implica que no existe un modelo masculino universal y válido para cualquier lugar, época, clase social, edad, raza, orientación sexual. Más bien existe una diversidad heterogénea de 
identidades masculinas y de maneras de ser hombre en nuestra sociedad. Ramírez (1999), expresa que lo masculino y lo femenino no constituyen una realidad separada del sujeto, son para este autor una construcción cultural cuyo fundamento no es biológico, esto a pesar de tener como base las diferencias biológicas. Es pues construido, organizado y regulado por un sistema de creencias, reglas y expectativas. Lomas (2004), por su parte expresan que la masculinidad es una construcción social en la medida en que a los hombres se les asigna socialmente ideologías y tareas que influyen en las diversas y múltiples formas de ser y sentirse hombres.

Sin embargo, existen una serie de referentes en cuanto a lo que debe ser lo masculino, una versión dominante de la masculinidad. La versión dominante de la masculinidad, de acuerdo a Badinter (1993), no constituye una esencia, sino una ideología de poder y de opresión que tiende a justificar la dominación masculina. Connell (1997) se refiere a esta versión dominante como masculinidad hegemónica definiéndola así: "la configuración de práctica genérica que encarna la respuesta corrientemente aceptada al problema de la legitimidad del patriarcado, la que garantiza (o se toma para garantizar) la posición dominante de los hombres y la subordinación de las mujeres" (pág.39). Según Donalson (1993) la masculinidad hegemónica también es misógina y homofóbica. La masculinidad hegemónica establece un régimen de poder y privilegios que excluyen a las mujeres y a los hombres que no cumplen con las expectativas de la construcción social del género.

Ramírez y García Toro (2002) destacan que los hombres que personifican la masculinidad hegemónica deben demostrarlo, probarlos en el porte, el manejo de sus cuerpos, el tono de sus voces y en el proceder. En cuanto al manejo del cuerpo, Solano Castillo (2007) expresa que el "ser hombre", tiene que tomar en cuenta el cuerpo. Es necesario tomar en cuenta la contextura física, los movimientos corporales, la utilidad social que el cuerpo masculino brinda al relacionarse con los demás y la comparación de ese cuerpo con otros cuerpos. El cuerpo tiene entonces una serie de sentidos, significados y percepciones en el campo de lo social, y muy directamente en la construcción social de identidades masculinas.

Uno de los argumentos de Pope et al. (2000) es que muchas de las inseguridades e insatisfacción de los hombres con relación a sus cuerpos han surgido por la creciente igualdad y equidad entre hombres y mujeres en muchos aspectos. Paralelo al proceso histórico de camino a la equidad y justicia entre los géneros, surgieron cambios en las actitudes culturales hacia el cuerpo masculino. Los hombres comenzaron a perder gradualmente sus identidades tradicionales como protectores y proveedores. Con el declive de estas identidades masculinas tradicionales, es que la importancia del cuerpo masculino incrementa. El cuerpo va creciendo en importancia como una característica distintiva de la masculinidad. Solano Castillo (2007) indica que el cuerpo existe entre instituciones que contienen implicaciones y discursos de género. Estos discursos los compara con mapas cognitivos de las posibilidades y limitaciones que tiene el cuerpo 
en la sociedad. El cuerpo es pues un elemento central para alcanzar la definición de lo que es ser un hombre completo. Las posibilidades de ese cuerpo deben llevarse a la muscularidad, cuanto más grande en tamaño mejor.

El cuerpo musculoso es un símbolo de distinción masculina. El cuerpo masculino con desproporcionadas formas de masa muscular, está vinculado a los ideales y visiones sociales de lo que es ser un hombre. Debe ser fuerte, poderoso, eficaz y dominante. Connell (1995) y Segal (1990), coinciden en que la masculinidad se asocia con cierto tipo de piel, ciertas posturas, formas de moverse, con la eficiencia física y sobre todo con el endurecimiento de los músculos, los cuales adquieren un nuevo significado. Un cuerpo desarrollado con músculos encarna los atributos centrales de la construcción social de la masculinidad hegemónica, que afecta e implica a todas las variantes de las masculinidades (Johnston, 2001; Mishkind, Rodin, Siberstein \& Striegel, 1986). Con relación a este planteamiento Randall et al. (1992), (citado en Pope et al. 2000), expresa que tener un cuerpo musculoso es una forma de irradiar poder y masculinidad. Un cuerpo musculoso es un intento de los hombres por restaurar los sentimientos masculinos de auto-control y dignidad (Gillet y White, 1992). Según White y Gillet (1994), a través del desarrollo de un cuerpo musculoso mediante el levantamiento de pesas y otras prácticas y conductas asociadas, los hombres intentan reafirmar la versión patriarcal convencional de la masculinidad.

Tomando como base la revisión de literatura y las consideraciones teóricas expuestas anteriormente realice una investigación para obtener el grado de Maestría en Trabajo Social en la Universidad de Puerto Rico que tuvo como propósitos principales: explorar la percepción en torno a la imagen corporal en hombres que asisten a gimnasios y conocer la motivación de hombres que acuden al gimnasio a realizar ejercicios.

\section{Diseño metodológico}

La investigación utilizó un diseño exploratorio de corte cuantitativo. El investigador realizó un protocolo de investigación autorizado por el Comité Institucional para la Protección de Sujetos Humanos en la Investigación (CIPSHI) del Recinto de Río Piedras de la Universidad de Puerto Rico. Como proyecto académico la investigación contó con la dirección de la Dra. Nilsa M. Burgos Ortiz, Catedrática de la Escuela Graduada de Trabajo Social Beatriz Lassalle. La población que se estudió fueron hombres mayores de 21 años de edad, que asistían a gimnasios a ejercitarse. La muestra de esta población estuvo constituida por 20 hombres. La selección de participantes se llevó a cabo por medio de muestreo no probabilístico, por disponibilidad.

Para recopilar la información de esta investigación se utilizó una planilla que construyó y administró el investigador, la misma incluyó preguntas cerradas y abiertas. También se utilizaron las preguntas del "Cuestionario del Complejo de Adonis" desarrollado por Pope et al. (2000). La planilla se dividió en tres partes principales: datos 
sociodemográficos, Cuestionario del Complejo de Adonis y preguntas sobre conductas asociadas a la Dismorfia Muscular. Los datos se analizaron por medio de procedimientos estadísticos descriptivos. Se utilizó el programa "Statistical Package for Social Sciencies" (SPSS) versión 16.0, mediante el cual se obtuvieron frecuencias y por cientos.

\section{Resumen de los Hallazgos}

Los hallazgos de la investigación establecen una tendencia en los hombres entrevistados a tener una percepción de su imagen corporal con diversos grados de insatisfacción que a su vez los motiva para realizar ejercicios en el gimnasio. Se encontraron grados de insatisfacción con la imagen corporal en su totalidad y en partes específicas del cuerpo. De igual forma percepciones que parecen estar acorde con la insatisfacción.

\section{Datos Socio-demográficos}

La mitad (50\%) de los hombres entrevistados se encontraban entre las edades de 31 a 40 años de edad. La gran mayoría (70\%) de los hombres entrevistados informó que su estado civil era la soltería. El nivel de preparación académica fue muy alto. Cerca de la mitad (45\%) informó tener educación graduada como: maestría (25\%),doctorado (15\%) y Juris Doctor $(5.0 \%)$. Los ingresos económicos de los entrevistados fueron relativamente altos. Más de una tercera parte (35\%) devengaba $\$ 50,000$ dólares o más anualmente. Un $20 \%$ tenía ingresos anuales entre $\$ 30,000$ a $\$ 50,000$.

\section{Cuestionario del Complejo de Adonis}

La segunda parte de la planilla era el Cuestionario del Complejo de Adonis, el cual fue creado con el propósito de medir las señales de peligro con relación a la Dismorfia Muscular. El cuestionario se compone de 13 preguntas que exploran las preocupaciones que tienen los hombres en torno a su imagen corporal. El cuestionario preguntó específicamente sobre formas en cómo preocupaciones, inquietudes y conductas asociadas a la percepción de la imagen corporal pueden afectar la vida diaria de los hombres. Cada pregunta contiene tres alternativas para contestar identificadas con las letras a, b, c. Las alternativas tienen un valor de 0 punto (a), 1 punto (b) y 2 puntos (c).

Se obtiene una puntuación de entre 0 y 39 puntos, si se obtienen puntuaciones de 19 puntos en adelante demuestran una grave preocupación por como perciben su imagen corporal. Los resultados se interpretan considerando si el participante obtuvo entre 0 a 9 puntos, tiene preocupaciones menores relativas a su apariencia corporal. Si obtiene entre 10 y 19 puntos se considera que el participante tiene una forma moderada de la Dismorfia Muscular. De 20 a 29 puntos los problemas de imagen corporal en esta persona pueden ser un problema que afecte su salud física y mental. De 30 a 39 puntos implican un problema agudo de Dismorfia Muscular, los investigadores e investigadoras 
sugieren intervención urgente de un profesional de la salud mental que conozca sobre el tema. Los resultados obtenidos reflejan que la mayoría de los participantes (55\%) tienen una preocupación leve por aspectos relativos a su apariencia corporal. Un $40 \%$ de los entrevistados tiene una preocupación moderada. Uno solo de los participantes evidenció una preocupación grave.

En la figura número 1, podemos observar las puntuaciones obtenidas por los 20 participantes del estudio.

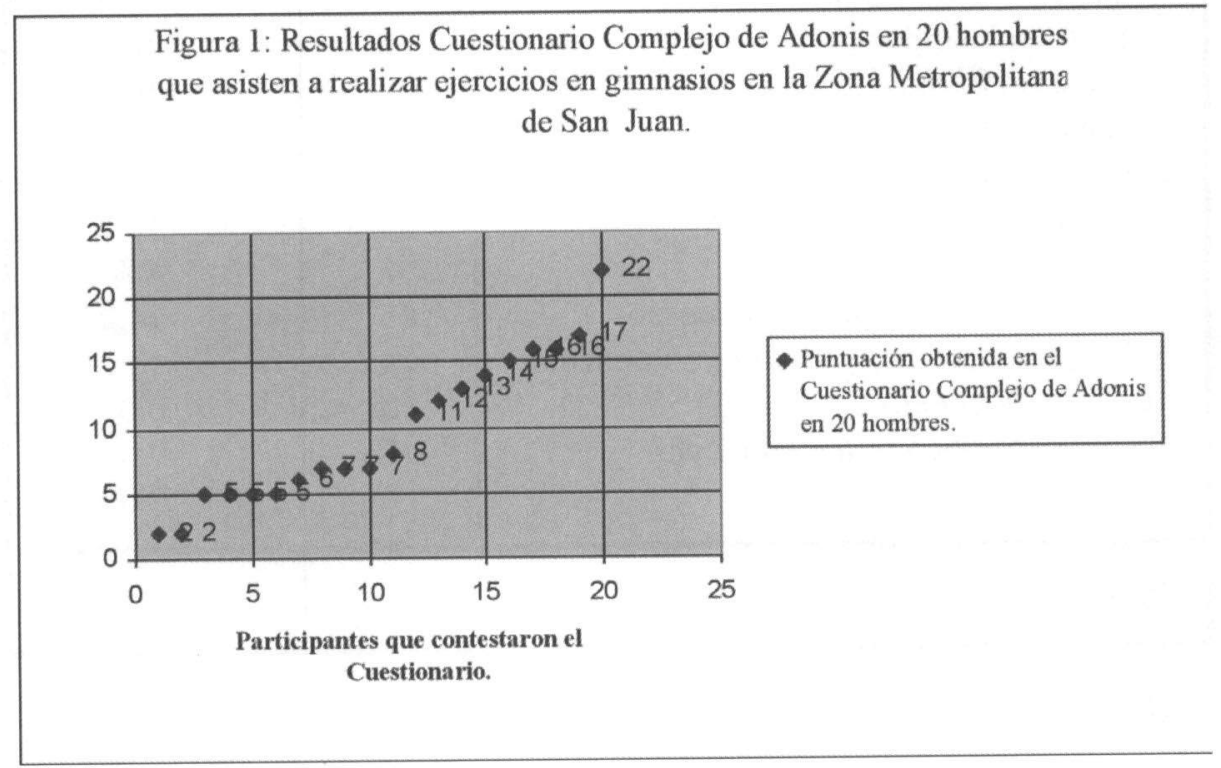

\section{Motivación para realizar ejercicios}

Las motivaciones para realizar ejercicios seleccionadas por los participantes con mayor número respuestas fueron: estar en forma (14.2\%), cuidar la salud (14.2\%), mejorar apariencia del cuerpo $(14.2 \%)$, reducir el estrés y mejorar la autoestima $(13.2 \%)$, mejorar condición física $(13.2 \%)$, ganar masa muscular $(10.4 \%)$. 
En la Tabla a continuación, mostramos los resultados en frecuencias y por ciento de las respuestas sobre motivación para realizar ejercicios en gimnasios.

\section{Tabla I - Frecuencias y por cientos para Motivación para realizar ejercicios en gimnasios}

\begin{tabular}{|l|c|c|}
\hline Motivación & $\mathrm{n}$ & $\%$ \\
\hline 1. Ganar masa muscular & 11 & 10.4 \\
2. Entretenimiento & 6 & 5.7 \\
3. Estar en forma & 15 & 14.2 \\
4. Bajar de peso & 10 & 9.4 \\
5. Cuidar su salud & 15 & 14.2 \\
6. Mejorar su condición Física & 14 & 13.2 \\
7. Ganar seguridad & 5 & 4.7 \\
8. Mejorar apariencia de su cuerpo & 15 & 14.2 \\
9. Reducir el estrés y mejorar autoestima & 14 & 13.2 \\
10. Otra respuesta & 0 & 0.0 \\
11. No especificó otra Respuesta & 0 & 0.0 \\
12. No contestó & 0 & 0.0 \\
\hline Totales & 105 & 100,0 \\
\hline
\end{tabular}

\section{Grados de Insatisfacción con la Imagen Corporal}

Se encontraron grados de insatisfacción con la imagen corporal en su totalidad y en partes específicas del cuerpo: Tamaño Bíceps (55\%), Tamaño Cintura (60\%), Volumen del Pecho (55\%), Tonificación Abdominal (70\%), Peso Corporal (35\%), Constitución Muscular (50\%), Imagen Corporal en su totalidad (25\%).

\section{Utilización de Esteroides Anabólicos Androgénicos}

Solo cuatro (4) participantes expresaron haber utilizado estas sustancias. A los dieciséis participantes que contestaron que no habían utilizado esteroides anabólicos, se les pregunto si habían considerado utilizarlos. De éstos, 4 indicaron que lo consideraron, y los otros 12 indicaron que no.

\section{Percepciones en cuanto a la imagen corporal}

En lo relativo a la conciencia sobre la imagen corporal los hombres entrevistados vinculaban la percepción de su imagen a diferentes aspectos como la salud física y 
mental, el desarrollo de masa muscular, la alimentación ó dieta, peso corporal, características físicas, aspectos de interacción y éxito en las áreas social y laboral.

\section{Discusión e implicaciones para la práctica del Trabajo Social:}

La percepción en torno a la imagen corporal de los hombres entrevistados estuvo matizada por pensamientos, emociones y conductas que pudieran tener alguna relación con la insatisfacción de la imagen corporal. Los hombres entrevistados expresaron una variedad de respuestas a la pregunta relativa a cómo perciben su imagen corporal. Entre la variedad de respuestas encontradas los participantes percibían su imagen corporal con diversos pensamientos y emociones. Entre los hallazgos obtenidos se observa una preocupación de los participantes por no ser lo suficientemente magros y con volumen muscular. Desde la perspectiva de género esta preocupación podría estar asociada a que el cuerpo musculoso es un símbolo de distinción masculina.

De acuerdo a Arbinaga y Caracuel (2003) la insatisfacción con la imagen corporal se manifiesta en el trasfondo de las razones por las cuales los hombres acuden a los gimnasios a realizar ejercicios. Como un sujeto masculino, percibe o interpreta su apariencia, pudiera llevarlo a tener una insatisfacción con su cuerpo total o parcialmente. Como consecuencia lo puede llevar a realizar una amplia gama de actividades y conductas con la intención de solucionar esa insatisfacción.

En el ejercicio de la práctica del Trabajo Social, es indispensable revisar, mirar y reflexionar sobre los cimientos que dieron base a la creación y desarrollo de nuestra profesión. Sobre todo, aquel principio que nos remite a luchar por acercarnos a la justicia social. Existe poca información accesible en la literatura sobre la dimensión y condición masculina. Deberían existir más esfuerzos en la práctica de la profesión dirigidos a concienciar a los hombres sobre la construcción social de las masculinidades y los asuntos relacionados, como bien puede ser el caso de las distorsiones en la imagen corporal. Repasando la investigación específica sobre el Complejo de Adonis, podemos ver cómo afecta el desarrollo integral y expone a los hombres a asumir conductas de alto riesgo y el no poder disfrutar de una vida plena.

Debido a las implicaciones de la Dismorfia Muscular en aspectos de salud pública, los Trabajadores y Trabajadoras Sociales pueden aportar en el análisis desde aspectos sociales específicamente en como la estructura social patriarcal en que vivimos, estimula la construcción de identidades masculinas en la cual el cuerpo es referente central para la dominación y la violencia. Mediante el desarrollo de un cuerpo musculoso y magro con el levantamiento de pesas y otras prácticas y conductas asociadas, los hombres intentan reafirmar la versión patriarcal convencional de la masculinidad.

El Trabajo Social puede hacer aportaciones en los aspectos sociales que afectan a los hombres con Dismorfia Muscular. El bienestar integral de estos hombres, es parte de 
esa justicia social a la que aspiramos. Los trastornos de imagen corporal en hombres, son relevantes para el Trabajo Social, en la medida que miremos a los hombres como sujetos con derechos a un bienestar social e integral. Nuestra aportación bien puede ser desde la intervención clínica directa, aportando protocolos y modelos de intervención. Asimismo en el trabajo interdisciplinario con otras ramas de las ciencias de la conducta y de las ciencias médicas.

Pérez, Valencia, Rodríguez \& Gempeler (2007) expresan que el reconocimiento de la condición por parte de los afectados, es un reto porque éstos se sienten saludables. Esta apariencia saludable y percepción personal de bienestar por parte de los hombres afectados hace apremiante la necesidad de parte del terapeuta de agudizar la observación, una aproximación cuidadosa, empática y sensible a su condición. En estos aspectos de intervención clínica los Trabajadores y las Trabajadoras Sociales cuentan con herramientas aprendidas y desarrolladas en su proceso formativo para lograr el abordaje de la situación con los afectados. La aportación desde la práctica directa puede ser amplia y dirigida a lograr la concienciación y posterior acción de estos hombres a fin de lograr bienestar, y por consiguiente una mejor calidad de vida.

De igual forma es pertinente a la investigación en Trabajo Social, en la medida que aportemos en la explicación de aspectos sociales y culturales que inciden en la construcción del Complejo de Adonis. Además de aumentar el conocimiento sobre la condición por medio de investigaciones enfocadas desde aspectos de intervención social y de las aportaciones que puede hacer el Trabajo Social en el ámbito individual, grupal e incluso comunitario.

La educación en Trabajo Social es responsable de contribuir a la formación de sus estudiantes sobre este nuevo problema masculino. La academia puede aportar a la explicación y entendimiento del problema, la intervención social e interdisciplinaria y la prevención en todas sus fases. Asimismo, es de importancia el conocimiento sobre los problemas que afectan a la población masculina para la intervención con familias donde hay niños, adolescentes, hombres jóvenes y adultos. A pesar de las nociones asociadas al control del poder que han tenido los hombres históricamente, en las últimas décadas hemos observado cómo puede ser una población vulnerable a diferentes situaciones. La educación en Trabajo Social, debe incluir esta nueva dimensión de los estudios asociados al género, propiciando y subvencionando investigaciones en el área, así como seminarios, talleres y jornadas de estudios sobre las masculinidades.

Sería recomendable que organizaciones de bienestar social relativas a la recreación y el deporte, brindarán orientación preventiva a los participantes de los deportes sobre la Dismorfia Muscular y sobre los efectos del uso de esteroides anabólicos androgénicos. El desarrollo de grupos de orientación y apoyo para personas en riesgo de trastornos relativos a la apariencia corporal y de servicios de prevención en la comunidad y en gimnasios. 


\section{Referencias}

American Psychiatric Association. (2000). Diagnostic and Statistical Manual of Mental Disorders DSM IV-TR (fourth edition). Washington D.C.: APA

Arbinaga, Félix y Caracuel, José Carlos. (2003). Aproximación a la Dismorfia Muscular. Cuadernos de Medicina Psicosomática y Psiquiatrica de enlace, 65, 7-15.

Badinter, Elizabeth. (1993). XY: La identidad masculina. Madrid: Alianza.

Baile Ayensa, José I. (2002). ¿Qué es la imagen corporal? Recuperado el 2 de febrero de 2008 de: www.uned.es/catudela/revista/n002/baile_ayensa.htm

Baile Ayensa, José I. (2003). Vigorexia y culto al cuerpo, alteraciones de la imagen corporal en hombres. Recuperado el 29 de enero de 2008 de http:// www.psiquiatría.com/artículos/tralimentacion/9732/.

Baile-Ayensa, José I.; Monroy Martínez, Karina E. y Garay Rancel, Fernando (2005). Alteración de la imagen corporal en un grupo de usuarios de gimnasios. Enseñanza e Investigación en Psicología, 10 (1), 161-169.

Bergstrom, Rochelle L.; Neighbors, Clayton. (2006). Body Image Disturbance and The Social norms approach: An integrative review of literature. Journal of Social and Clinical Psychology, 25 (9), 975-1000.

Bonan, Claudia \& Guzmán, Virginia. (s.f.); Aportes de la teoría de género a la comprensión de las dinámicas sociales y los temas específicos de asociatividad y participación, identidad y poder. Recuperado el 29 de enero de 2008 de: http://www.isiscl//refdebates/aporte.pdf.

Connell, Robert W. (1995). Masculinities. Berkley and Los Angeles: University of California Press.

Connell, Robert W. ( 1997). Gender \& power. California: Stanford University Press.

Donaldson, M. (1993). What is Hegemonic Maculinity?. Theory and Society, 22, 643-657.

Facchini, Mónica. (2006). La imagen corporal en la adolescencia ¿es un tema de varones? Pediatría práctica, 104 (2), 177-184. 
Gillet, James \& White, Phillip. (1992). Male bodybuilding and the reassertion of egemonica masculinity: A critical feminist perspectiva. Play and Culture, 5 (4), 358-369.

Irigoyen-Coll, Cristina. (2005). Esteroides y percepción del riesgo. Recuperado el 14 de febrero de 2008, de: www.sendonavar.gov.ar/images/novedades/biblioteca/ coll\%esteroides.pdf

Johnston, Jessica R. (2001). The American Body in Context: An Anthology. Wilmington: Scholarly Resources Inc.

Kanayama, Gen; Pope, Harrison G.; Cohane, Geoffrey \& Hudson James I. (2003). Risk Factors for anabolic-androgenic steroid use among weightlifters: a case control study. Drug and alcohol dependence, 71, 77-86.

Leit, Richard A.; Pope, Harrison G. \& Gray, James. (2001). Cultural Expectations of muscularity in men: the evolution of Playgirl centerfolds. International Journal of eating disorders, 29, 90-93.

Leit, Richard A.; Gray, James \& Pope, Harrison G. (2002). The media's representation of the ideal male body: a cause for muscular dysmorphia? International Journal of eating disorders, 31, 334-338.

Lerner, Gerda. (1990). La Creación del Patriarcado. Barcelona: Editorial Crítica.

Lomas, Carlos. (2004). Los Chicos también lloran: Identidades masculinas, igualdad entre los sexos y coeducación. Barcelona, España: Paidós.

Mishkind, Marc E.; Rodin, Judith; Silberstein, Lisa R. \& Striegel-Moorre, Ruth. (1986). The Embodiment of masculinity: Cultural, psychological, and behavioral dimensions. American Behavioral Scientist, 29, 545-562.

Olivardia, Roberto; Pope, Harrison G. \& Hudson, James I. (2000). Muscle dysmorphia in male weightlifters: a case control study. American Journal of Psychiatry, 157, 1291-1296.

Pérez Restrepo, Victoria; Valencia, María Nelly; Rodríguez, Maritza y Gempeler, Juanita. (2007). Acerca de un caso de dismorfia muscular y abuso de esteroides. Revista Colombiana de Psiquiatría, 36 (1), 154-164.

Pope, Harrison G.; Gruber, Amanda J.; Choi, Phillip; Olivardia, Roberto \& Phillips, Katherine. (1997). Muscle dysmorphia. An underrecognized form of body dismorphic disorder. Psychosomatic, 39(6), 548-557. 
Pope, Harrison G.; Katz, David L. \& Hudson, James I. (1993). Anorexia nervosa and reverse anorexia among 108 male bodybuilders. Comprehensive Psychiatry, 34(6), 406-409.

Pope, Harrison G. Jr.; Olivardia, Roberto; Gruber, Amanda \& Borowiecki, John. (1999). Evolving ideals of male body image as seen through action toys. International Journal of eating disorders, 26 (1), 65-72.

Pope, Harrison G., Jr; Phllips, Katherine \& Olivardia, Roberto. (2000). The Adonis Complex: The secret crisis of male body obsession. New York: The Free Press.

Ramírez, Rafael. (1999). Dime capitán: Reflexiones sobre la masculinidad .(2da ed.). Río Piedras: Ediciones Huracán.

Ramírez, Rafael y García Toro, Víctor I. (2002). Masculinidad hegemónica, sexualidad y transgresión. CENTRO Journal of the Center for Puerto Rican Studies, XIV (I), 4-25.

Román Tirado, Félix; González Armenteros Juan J.; Fernández Bauzó, Edwin; Cruz Díaz, Edwin \& Ávila Rodríguez, Marviliz. (2003). Masculino que Ninguno: una perspectiva sociopersonal del género, el poder y la violencia. San Juan, Puerto Rico: Colectivo Ideologías y Vivencias de los Géneros.

Rutsztein, Gullermina; Casquet, Alejandra; Leonardelli, Eduardo; López, Pablo; Macchi, Marina; Marola, Maria Elena \& Redondo Gisela. (2004). Imagen corporal en hombres y su relación con la dismorfia muscular. Revista Argentina de Clínica Psicológica, 13 (2), 1-20.

Scott, Joan. (1996). El género, una categoría útil para el análisis histórico. En Marta Lamas (editora), El género: la construcción cultural de la diferencia sexual (265-302). México: Porrúa-Pueg.

Solano Castillo, Luis. (2007). El Cuerpo. En Víctor García-Toro, Rafael Ramírez y Luis Solano Castillo, Los Hombres no lloran. Ensayos sobre las masculinidades (19-63). San Juan: Ediciones Huracán.

Segal, Lynne. (1990). Slow motion: Changing masculinities, changing men. New Jersey: Rutgers University Press.

White, Phillip \& Gillet, James. (1994). Reading the muscular body: A critical decoding of advertisements in Flex Magazine. Sociology of Sport Journal 1 (4), 18-39. 
, 